\title{
ACHADOS NA RADIOGRAFIA PANORÂMICA INDICAM TOMOGRAFIA COMPUTADORIZADA NO PRÉ-OPERATÓRIO DE TERCEIRO MOLAR INFERIOR: RELATO DE CASO
}

\author{
PANORAMIC RADIOGRAPHY FINDINGS INDICATE COMPUTED TOMOGRAPHY IN \\ MANDIBULAR THIRD MOLAR PRESURGICAL EVALUATION: CASE REPORT
}

\author{
Thallita Mariano FERRAZ1; Luciano Sandoval CARNEIRO²; Juliana STECKE³; Nádia RAYES4; Giselle Barbosa de OLIVEIRA ${ }^{5}$ \\ 1 - Acadêmica do Curso de Odontologia, Universidade Paulista - UNIP, Brasília, DF, Brasil. \\ 2 - Professor Adjunto de Radiologia no Curso de Odontologia, Universidade Paulista - UNIP, Brasília, DF, Brasil. Cirurgião-dentista, Radiologista, \\ Secretaria de Estado de Saúde do Distrito Federal, Brasília, DF, Brasil. \\ 3 - Cirurgiã-dentista, Radiologista, Clínica Particular, Indaiatuba, SP, Brasil. \\ 4 - Cirurgiã-dentista, Radiologista, Clínica Particular, Florianópolis, SC, Brasil. \\ 5 - Cirurgiã-dentista, Radiologista, Clínica Particular, Trindade, GO, Brasil.
}

\section{RESUMO}

Os terceiros molares inferiores apresentam-se frequentemente retidos e suas raízes podem estar envolvidas pelo canal da mandíbula, representando risco de injúria nervosa durante a exodontia. Na avaliação pré-cirúrgica desses dentes, comumente utiliza-se a radiografia panorâmica, que muitas vezes evidencia a sobreposição das imagens das raízes dentárias sobre o canal da mandíbula, com possibilidade de proximidade entre estas estruturas. Esses casos geram dúvidas ao profissional quanto à necessidade de indicar uma tomografia computadorizada. Na literatura têm sido pesquisados achados na radiografia panorâmica que indicam maior risco de proximidade entre tais estruturas, justificando a indicação do exame seccional. Entre esses achados, destacam-se: escurecimento radicular, interrupção da linha radiopaca do canal, desvio do canal, deformação da raiz, estreitamento do canal, estreitamento radicular, escurecimento e ápice bífido da raiz. A presença de um ou mais desses sinais em radiografia panorâmica indica provável proximidade entre as raízes do terceiro molar e o canal mandibular. O objetivo desse trabalho foi relatar um caso clínico de avaliação do dente 48 previamente à exodontia, envolvendo uma radiografia panorâmica, que revelou dois desses sinais apontados na literatura. Posteriormente, foi obtida tomografia computadorizada por feixe cônico (TCFC), que evidenciou envolvimento de ambas as raízes do dente 48 pelo canal da mandíbula. Concluiu-se que a TCFC foi bem indicada, devendo ser recomendada quando a radiografia panorâmica evidenciar um ou mais dos sinais indicativos de proximidade entre raízes do siso e o canal da mandíbula, contribuindo para a decisão na rotina clínica e para evitar complicações pós-operatórias.

Palavras-chave: Terceiro molar; Nervo alveolar inferior; Radiografia panorâmica; Tomografia computadorizada.

\section{INTRODUÇÃO}

A remoção de terceiros molares inferiores é um dos procedimentos cirúrgicos mais comuns na Odontologia ${ }^{1-3}$ e requer um planejamento cuidadoso para evitar acidentes e complicações como a parestesia do nervo alveolar inferior ${ }^{2,3}$. Na fase pré-operatória desses dentes, é indispensável uma avaliação criteriosa tanto do posicionamento desses elementos quanto da relação dos mesmos com o canal da mandíbula. Para essa finalidade, na rotina clínica, a radiografia panorâmica é frequentemente utilizada ${ }^{1,3,4}$.

Entretanto, essa modalidade de imagem é bidimensional, não permitindo avaliar a relação entre o terceiro molar e o canal da mandíbula no sentido vestíbulo-lingual ${ }^{2,4}$. Muitas vezes, a radiografia panorâmica revela sobreposição das imagens dessas estruturas. Assim, em casos de maior risco de proximidade entre tais componentes, recomenda-se o emprego da tomografia computadorizada, cujas imagens revelam o volume escaneado em vários planos anatômicos, permitindo checar com precisão a relação entre o dente siso e o canal mandibular. Inclusive, a utilização desse exame seccional tem ampliado na Odontologia, com o advento da tomografia computadorizada por feixe cônico (TCFC), cujos aparelhos geram imagens com maior resolução espacial e, na maioria das situações, expõem o paciente a uma menor dose de radiação, se comparados aos tomógrafos espirais ${ }^{3}$.

Porém, para assegurar ao paciente a menor exposição possível à radiação ionizante, bem como menor custo, deve-se selecionar adequadamente os casos que requerem TCFC. Na literatura têm sido pesquisados achados na radiografia panorâmica que podem indicar maior risco de proximidade entre raízes dos sisos e o canal mandibular e, portanto, contribuir na indicação da TCFC. Entre esses achados, destacam-se: escurecimento radicular ${ }^{2,3,5,6}$, interrupção da linha radiopaca do $\operatorname{canal}^{2,3,5,6}$, desvio do canal ${ }^{7,8}$, deformação da raiz ${ }^{9}$, estreitamento do canal $^{2,3,5}$, estreitamento radicular ${ }^{9}$, escurecimento e ápice bífido da raiz ${ }^{9}$. 
O objetivo deste trabalho foi relatar o caso clínico de uma paciente, cuja radiografia panorâmica revelou sinais de possível proximidade entre um terceiro molar inferior retido e o canal mandibular, bem como abordar a importância da tomografia computadorizada para situações dessa natureza.

\section{RELATO DE CASO}

Paciente F.G.F., 21 anos, gênero feminino, compareceu a uma clínica privada de Radiologia Odontológica, na cidade de Indaiatuba- SP. Portava um formulário solicitando uma radiografia panorâmica dos maxilares para avaliação de terceiros molares. Relatou que o cirurgião-dentista responsável pelo seu tratamento recomendou a exodontia desses dentes, considerando a deficiência de espaço para o irrompimento dos mesmos na cavidade bucal e a ocorrência de episódios recorrentes de processo inflamatório da mucosa adjacente a alguns deles.

A radiografia panorâmica (Figura 1) evidenciou a retenção dos dentes 38 e 48, ambos em posição mésio-angular, e dos dentes 18 e 28 , ambos na vertical. O exame também revelou a sobreposição das imagens das raízes dos dentes 38 e 48 com o canal da mandíbula.

Considerando-se a presença de dois sinais radiográficos sugestivos de proximidade entre canal da mandíbula e raízes dos terceiros molares inferiores (vide Figura 1), tanto no dente 38 quanto no dente 48 , foi recomendada tomografia computadorizada por feixe cônico (TCFC) desses dois sítios para melhor detalhe diagnóstico.

Como a paciente optou por extrair inicialmente apenas o dente 48, a TCFC foi adquirida somente nesse sitio. As imagens tomográficas evidenciaram o envolvimento de ambas as raízes do dente 48 pelo canal mandibular (Figuras 2 e 3).

\section{DISCUSSÃO}

Há um consenso entre Deshpande et al..$^{5}$ (2013), Matzen et al..$^{3}$ (2015) e Tantanapornkul et al. ${ }^{10}(2016)$, que consideram a radiografia panorâmica confiável na maioria dos casos de avaliação pré-operatória de terceiro molar inferior. No entanto, Neves et al. ${ }^{7}$ (2012) relatam que essa modalidade de exame radiográfico não possui uma alta precisão diagnóstica para essa finalidade. Além disso, Andakani et al. ${ }^{11}$ (2011) consideram que a radiografia panorâmica apresenta limitações por revelar as informações em apenas duas dimensões, faltando a profundidade. Assim, nos casos em que a panorâmica evidencia possibilidade de proximidade entre o dente siso e o canal da mandíbula, esses autores recomendam o emprego da TCFC associada para o planejamento pré-operatório. Esses achados são corroborados por estudos mais recentes, como os de Haranda et al. ${ }^{8}$ (2015), Matzen et al. ${ }^{12}$ (2016) e Winstanley et al. ${ }^{2}$ (2018).

Porém, deve-se considerar que a dose de radiação envolvida na TCFC é superior àquela utilizada na radiografia panorâmi$\mathrm{ca}^{3}$. Assim, a TCFC é justificável em casos bem selecionados, nos quais contribuirá decisivamente para reduzir o tempo cirúrgico e evitar o desconforto das complicações pós-operatórias como a parestesia ${ }^{2}$.

Para contribuir na seleção adequada dos casos que requerem TCFC, na literatura vários estudos têm investigado achados nas radiografias panorâmicas que sugerem maior risco de proximidade entre o terceiro molar e o canal da mandíbula. Conforme

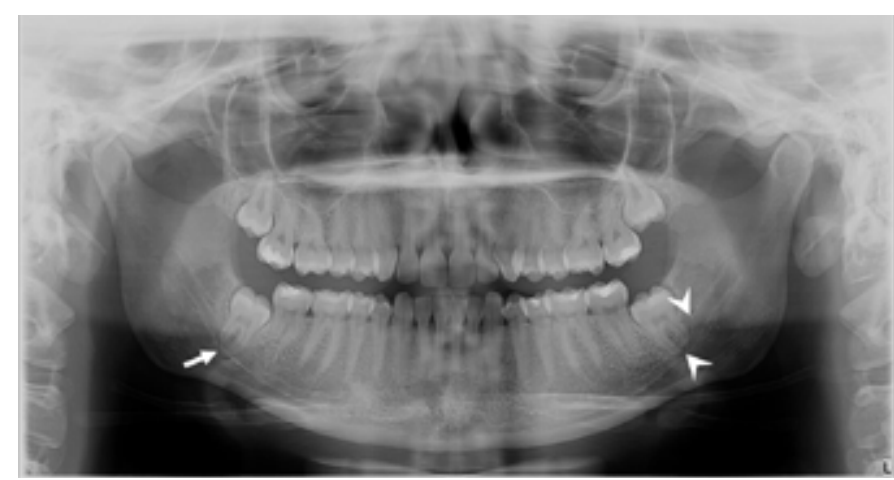

Figura 1 - Radiografia panorâmica: evidência de retenção dos dentes 38 e 48; escurecimento do ápice radicular e o desvio das raízes do dente 48 (seta); no sitio do dente 38 nota-se escurecimento no ápice radicular e desvio do canal mandibular (cabeças de seta).
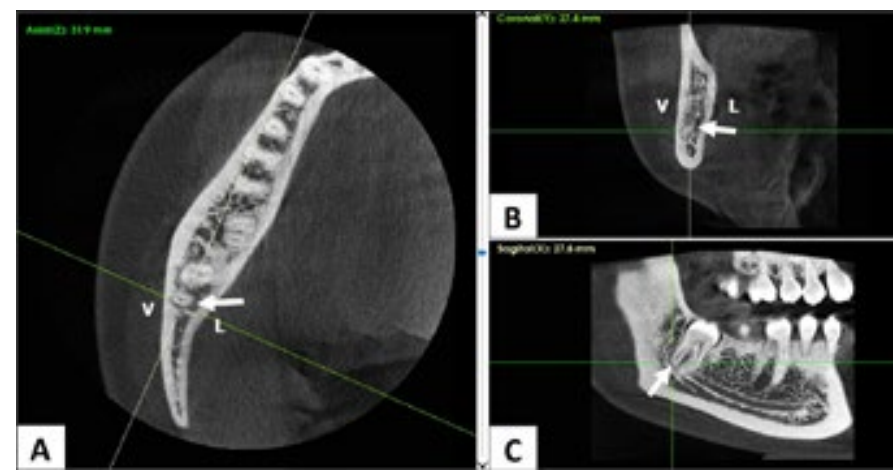

Figura 2 - TCFC: cortes axial (A), coronal (B) e sagital (C) revelam envolvimento do ápice da raiz distal do dente 48 pelo canal da mandíbula (vide setas). V: cortical óssea vestibular; L: cortical óssea lingual.
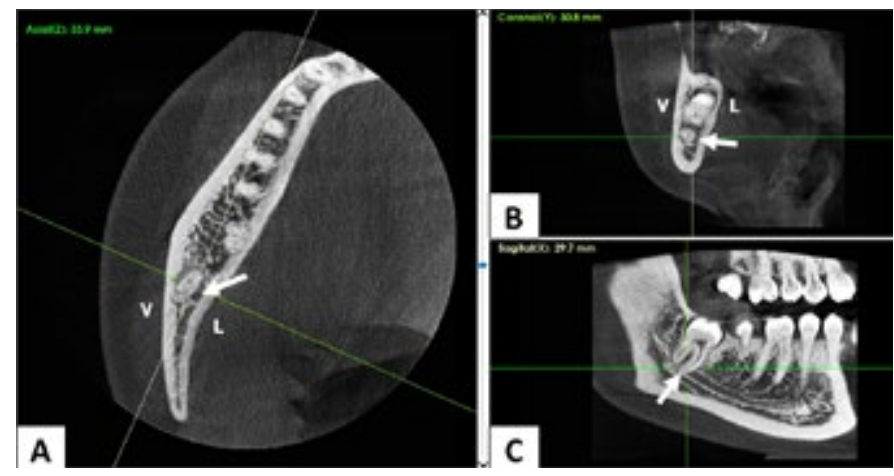

Figura 3 - TCFC: cortes axial (A), coronal (B) e sagital (C) revelam envolvimento do ápice da raiz mesial do dente 48 pelo canal da mandíbula (vide setas). V: cortical óssea vestibular; L: cortical óssea lingual.

Winstanley et al. ${ }^{2}$ (2018), Deshpande et al..$^{5}$ (2013), e Matzen et al. ${ }^{3}$ (2015), os principais sinais encontrados nas radiografias panorâmicas são: 1) escurecimento dos ápices radiculares; 2) interrupção da linha radiopaca da cortical superior do canal da mandíbula; 3) estreitamento do canal mandibular. Essas conclusões são confirmadas no estudo de Shanidi et al. ${ }^{6}$ (2013), que detectaram o escurecimento dos ápices radiculares ou a interrupção da linha radiopaca da cortical superior do canal mandibular em radiografias panorâmicas de pacientes cujos dentes sisos estavam em contato com o canal da mandíbula. 
Neves et al..$^{7}$ (2012) e Haranda et al. ${ }^{8}$ (2015) acrescentam um outro achado: o desvio do canal mandibular. Essa particularidade foi evidenciada nas radiografias panorâmicas de pacientes que manifestaram exposição do nervo alveolar inferior após a exodontia dos terceiros molares.

Jerjes et al. ${ }^{9}$ (2009) acrescentam mais três achados na radiografia panorâmica que sugerem contato entre dente siso e o canal mandibular: 1) estreitamento radicular; 2) desvio da raiz; 3) escurecimento e ápice bífido da raiz.

No presente relato, dois desses achados previamente abordados na literatura foram identificados na radiografia panorâmica no sítio do dente 48. Havia o escurecimento do ápice radicular e o desvio das raízes. Considerando-se que a presença de um ou mais desses sinais torna recomendável o exame seccional ${ }^{3,5}$, no presente estudo foi realizada a TCFC, que evidenciou o envolvimento de ambas as raízes do dente 48 pelo canal mandibular. Esses achados corroboram informações de estudos prévios ${ }^{2,3,5,6,9}$. Na mesma radiografia panorâmica do presente relato, no sítio do dente 38 retido, foram identificados escurecimento no ápice radicular e desvio do canal da mandíbula, sinais que justificam exame da região por TCFC. Entretanto, a paciente optou por adiar a extração desse dente.

Considerando que pode haver relação de proximidade entre raízes de dentes sisos e canal mandibular, o nervo alveolar inferior está sujeito a injúrias pós-operatórias. Em relação à parestesia permanente, na literatura há relatos de baixa incidência, entre $0,1 \%$ e $1.1 \%{ }^{1}$. No estudo de Korkmaz et al. ${ }^{13}$ (2017), envolvendo a extração de 139 terceiros molares inferiores cujas raízes estavam sobrepostas ao canal da mandíbula na radiografia panorâmica, não houve relato de parestesia permanente. Reportaram parestesia temporária em $16,4 \%$ dos casos operados utilizando apenas radiografia panorâmica no pré-operatório. Contudo, mesmo que a parestesia não seja permanente, o desconforto pode ser evitado com o uso da TCFC, uma tecnologia acessível atualmente.

\section{CONCLUSÕES}

A radiografia panorâmica pode ser suficiente na maioria dos casos de planejamento pré-operatório para exodontia de terceiros molares inferiores. A TCFC deve ser indicada quando um ou mais achados na radiografia panorâmica sugerem proximidade entre as raízes do terceiro molar e o canal mandibular. A interrupção da linha radiopaca do canal da mandíbula, escurecimento dos ápices radiculares de terceiros molares e desvio do canal mandibular em radiografias panorâmicas foram associados a um maior risco de lesão do nervo alveolar inferior. No presente relato de caso, dois achados da radiografia panorâmica contribuíram para indicar a TCFC, que evidenciou envolvimento das raízes do terceiro molar pelo canal mandibular.

\section{REFERÊNCIAS}

01. Petersen LB, Vaeth M, Wenzel A. Neurosensoric disturbances after surgical removal of the mandibular third molar based on either panoramic imaging or cone beam CT scanning: A randomized controlled trial (RCT). Dentomaxillofacial Radiol. 2016; 45(2):1-9.

02. Winstanley KL, Otway LM, Thompson L, Brook ZH, King N, Koong $\mathrm{B}$, et al. Inferior alveolar nerve injury: Correlation between indicators of risk on panoramic radiographs and the incidence of tooth and mandibular canal contact on cone-beam computed tomography scans in Western Australian population. J Invest Clin Dent. 2018; 9(3): 1-6.

03. Matzen LH, Wenzel A. Efficacy of CBCT for assessment of impacted mandibular third molars: A review based on a hierarchical model of evidence. Dentomaxillofacial Radiol. 2015; 44(1): 1-11.

04. Gu L, Zhu C, Chen K, Liu X, Tang Z. Anatomic study of the position of the mandibular canal and corresponding mandibular third molar on cone-beam computed tomography imagens. Surg Radiol Anat. 2018; 40(6): 609-614.

05. Deshpande P, Mahima V, Patol GK. Proximity of Impacted Mandibular Third Molars to the Inferior Alveolar Canal and it Radiographic Predictors: A panoramic Radiographic Study. J Maxillofac Oral Surg. 2013; 12(2): 145-151.

06. Shanidi S, Zamiri B, Bronoosh P. Comparison of panoramic radiography with cone beam $\mathrm{CT}$ in predicting the relationship of the mandibular third molar roots to the alveolar canal. Imaging Sci Dent. 2013; 43(2): 105-109.

07. Neves FS, Souza TC, Almeida SM, Haiter F, Freitas DQ, Bóscolo FN. Correlation of panoramic radiography and cone beam CT findings in the assessment of the relationship between impacted mandibular third molars and the mandibular canal. Dentomaxillofacial Radiol. 2012; 41(7): 553-557.

08. Haranda N, Subash BV, Matsuda Y, Seki K, Kapila R, Ishikawa N, et al. Characteristic Findings on Panoramic Radiography and Conebeam CT to Predict Paresthesia after Extraction of Impacted Third Molar. Bull Tokyo Dent Coll. 2015; 56(1): 1-8.

09. Jerjes W, Upile T, Kafas P, Abbas S, Rob J, McCarthy P, et al. Third molar surgery: the patient's and the clinician's perspective. Int Arch Med. 2009; 2(1): 32.

10. Tantanapornkul W, Marvin D, Prapaiphittayakun J, Phipatboonyarat $\mathrm{N}$, Julphantong W. Accuracy of panoramic radiograph in assessment of the relationship between mandibular canal and impacted third molars. The Open Dentistry Journal. 2016; 10: 322-329.

11. Andakani FE, Booshehri MZ, Behniafar B. Evaluation of the distortion rate of panoramic and periapical radiographs in erupted third molar inclination. Iran J Radiol. 2011; 8(1): 8-15.

12. Matzen L, Petersen L, Wenzel A. Radiographic methods used before removal of mandibular third molars among randomly selected general dental clinics. Dentomaxillofacial Radiol. 2016; 45 (4):1-7.

13. Korkmaz YT, Kayipmaz S, Senel FC, Atasoy KT, Gumrukcu Z. Does additional cone beam computed tomography decrease the risk of inferior alveolar nerve injury in high-risk cases undergoing third molar surgery? Does CBCT decrease the risk of IAN injury? Int J Oral Maxillofac Surg. 2017; 46(5): 628-635. 


\section{ABSTRACT}

Mandibular third molars are often intraosseous and their roots may be involved by mandibular canal, a risk situation of nerve injury during exodontia. For presurgical evaluation, panoramic radiography is widely used, so that the image often shows the overlapping of dental root images over the mandibular canal, with possible proximity between them. Then, the professional has doubts about the need to indicate a CT scan. In literature, researchers have investigated findings on panoramic radiography that indicate a greater risk of proximity between such structures, justifying the indication of the sectional examination. These findings include root darkening, interruption of white line of mandibular canal, canal deviation, root deformation, canal narrowing, root narrowing, browning and bifid apex of the root. The presence of one or more of these signs in panoramic radiography indicates probable proximity between lower third molar roots and mandibular canal. The aim of this study was to report a clinical case of a tooth 48 presurgical evaluation, involving a panoramic radiograph, which revealed two of these signs pointed out in the literature. Later, a cone beam computed tomography (CBCT) scan was performed, so that sectional images showed involvement of both roots of the tooth 48 by the mandibular canal. It was concluded that the CBCT was well indicated and should be recommended when the panoramic radiography evidences one or more of the signs indicating proximity between wisdom tooth roots and mandibular canal, contributing to the decision in clinical routine and to avoid postoperative complications.

KEYWORDS: Third molar; Inferior alveolar nerve; Panoramic radiography; Computed tomography.

\section{AUTOR PARA CORRESPONDÊNCIA}

Prof. Luciano Sandoval Carneiro

Universidade Paulista - Campus Brasília - Instituto de

Ciências da Saúde - Curso de Odontologia

SGAS Quadra 913, s/nº - Conjunto B - Asa Sul, Brasília - DF,

CEP 70390-130

Fone: (61) 2192-7000

E-mail: lucsanc@yahoo.com.br 